\title{
Alternative \& complementary treatment for pediatric inflammatory bowel disease
}

\author{
Ramit Magen, Ron Shaoul \\ Pediatric Gastroenterology and Nutrition Institute, Ruth Children's Hospital of Haifa, Rambam Medical Center, Faculty of Medicine, Technion, \\ Haifa, Israel \\ Contributions: (I) Conception and design: All authors; (II) Administrative support: None; (III) Provision of study materials or patients: None; (IV) \\ Collection and assembly of data: All authors; (V) Data analysis and interpretation: All authors; (VI) Manuscript writing: All authors; (VII) Final \\ approval of manuscript: All authors. \\ Correspondence to: Ron Shaoul, MD. Director, Pediatric Gastroenterology and Nutrition Institute, Ruth's Children's Hospital of Haifa, Rambam \\ Medical Center, POB 9602, Haifa 31096, Israel. Email: shaoul_r@012.net.il.
}

\begin{abstract}
Alternative medicine includes treatments that are not considered mainstream and is suggested to replace the accepted treatment, while complementary treatment is added to the conventional treatment. The estimated prevalence of their use in patients with inflammatory bowel diseases (IBD) is high, ranging between $21-60 \%$. This review summarizes the data on these treatments and their efficacy in the setting of IBD.
\end{abstract}

Keywords: Alternative therapy; complementary therapy; inflammatory bowel disease (IBD); children

Submitted Jun 29, 2019. Accepted for publication Aug 30, 2019.

doi: $10.21037 /$ tp.2019.09.02

View this article at: http://dx.doi.org/10.21037/tp.2019.09.02

\section{Introduction}

Inflammatory bowel diseases (IBD) are chronic auto inflammatory diseases that may involve the entire intestinal tract as in Crohn's disease (CD) or can be confined to the colon as in ulcerative colitis (UC).

Alternative medicine includes treatments that are not considered mainstream and is suggested to replace the accepted treatment, while complementary treatment is added to the conventional treatment. The estimated prevalence of their use in patients with IBD is high, ranging between $21-60 \%$ (1). This review summarizes the data on these treatments and their efficacy in the setting of IBD.

\section{Probiotics}

The current most accepted theory is that IBD develops due to an inappropriate inflammatory response to the gut microbiome in genetically susceptible individuals (2). It has also been demonstrated that the diversity of the microbiome in IBD patients is low compared to healthy controls (3).

A multicenter study that investigated the microbiome composition of treatment-naïve children with CD in comparison to healthy controls found that patients with $\mathrm{CD}$ had an increased level of aerobic and facultative anaerobes (Enterobacteriaceae, Fusobacteriaceae, Veillonellaceae and Pasteurellaceae). These may invade the epithelium and potentially exacerbate inflammation. Patients with CD have also been demonstrated to have decreased levels of obligate anaerobes (Bacteroides and Clostridiales). These organisms are considered beneficial to the host by increasing colonic Treg cells and downregulating intracellular signal transduction pathways, such as nuclear factor (NF)- $\mathrm{kB}$ and by producing short chain fatty acids, which are an important energy source for the colonic epithelium $(4,5)$. This shift is considered to be due to an increased oxygen concentration in the inflamed environment.

It has also been demonstrated that the use of antibiotics negatively affects the diversity of the microbiome, thereby contributing to the development of a dysbiotic state (4).

Probiotic microorganisms influence the intestinal microbiota by changing intestinal $\mathrm{pH}$, competing for nutrients, synthesis of antimicrobial compounds such as defensins, bacteriocins and blockage of bacterial adhesion 
and invasion to epithelial cells (6).

Whether probiotics have a positive effect in patients with CD is controversial. A recent systematic review on the effect of Saccharomyces bouladii in patients with IBD concluded that there is insufficient evidence to show that this probiotic is effective for the treatment of $\mathrm{UC}$ or $\mathrm{CD}$ due to the small number of trials ( 2 in CD and 1 in UC that showed a positive effect and 1 trial in CD that didn't find any effect in CD) (7).

Another systematic review with meta-analysis on the efficacy of probiotics in IBD, included 22 randomized controlled trials (RCTs) (8). There was no beneficial effect of probiotics in patients with $\mathrm{CD}$ for neither induction of remission or prevention of relapse of quiescent CD.

In patients with $\mathrm{UC}$, however, it seems that probiotics may have a positive effect on induction of remission and prevention relapse when the high-dose multi-bacterial agent VSL\#3 is administered (8). VSL\#3 is a probiotic preparation that is composed of 8 bacterial strains: $S$. thermophiles, L. paracasei, L. plantarum, L. acidophilus, $L$. delbrueckii, B. longum, B. breve, B. infantis.

In 2015, Fedorak et al. (9) evaluated the effect of VSL\#3 on patients with CD who were post small intestinal resection. The patients that were treated immediately after the surgery had lower inflammatory cytokines and lower recurrence rate compared to the patients who started treatment on the $90^{\text {th }}$ day post operation. However, the rate of severe recurrence and CD activity index were not significantly different between the two groups.

In a pediatric placebo controlled, double-blind study, 29 patients with UC were randomized to receive either placebo or VSL\#3 in addition to steroid therapy for induction of remission and mesalamine for maintenance (10). This study demonstrated that VSL\#3 is beneficial for induction of remission and for preventing relapse in 1-year follow-up. In another open label trial of children with mild to moderate UC, VSL\#3 taken for a period of 8 weeks in addition to the maintenance therapy or low steroid dose, induced endoscopic and clinical remission in $56 \%$ of patients, which was statistically significant (11). No adverse events were reported.

VSL\#3 has also been found to be effective in maintenance of remission in two double-blind, placebo controlled trials in adults with chronic or recurrent pouchitis $(12,13)$.

Lin et al. (14) also demonstrated that in mice gut, Lactobacillus rhamnosus $G G(L G G)$ prevents tumor necrosis factor alpha (TNF- $\alpha$ )-induced NF- $\mathrm{kB}$ activation and blocks inflammatory response in vitro and in vivo.
In a recent study, $L G G$ has been shown to reduce the expression of TNF- $\alpha$ and IL-17 in colonic biopsies taken from patients with UC (15). In vivo, the reduction of expression of TNF- $\alpha$ and IL-17 in these patients was dose dependent.

A systematic review and meta-analysis published in 2015, concluded that Escherichia coli Nissle 1917 is as effective as mesalazine in induction and maintenance of remission in UC patients (16).

In the European Crohn's and Colitis Organization (ECCO) and European Society for Pediatric Gastroenterology, Hepatology and Nutrition (ESPGHAN) guidelines, published in 2018, it is suggested that VSL\#3 or E. Coli Nissle 1917 may be used as an adjuvant therapy in mild UC for induction or maintenance of remission or as the sole treatment in mild UC with intolerance to 5-aminosalicylic acid (5-ASA) preparations. However, probiotics are not recommended in patients with $\mathrm{CD}$ due to lack of supporting evidence (17).

\section{Fecal microbiota transplantation (FMT)}

Several studies have indicated that an early alteration in the microbiome, either by the use of antibiotics or gastrointestinal (GI) infection, influences the risk to develop IBD.

FMT is an administration of processed feces from a donor into the patient's gut, hence increasing the diversity of the microbiome (18). Couturier-Maillard et al. (19) demonstrated that wild type (WT) mice that were cohoused with NOD2 mice, were more susceptible to develop colitis after dextran sodium sulfate (DSS) exposure. It was suggested that the NOD2 mice microbiome sensitized the mucosa to injury. Broad spectrum antibiotics and FMT decreased the risk of developing colitis in both WT and NOD2 mice.

One small pediatric study involving four patients with UC and one RCT in adults that were treated with FMT via nasogastric tube (NT) found no efficacy for NT administered FMT $(20,21)$.

In another small pediatric trial, 10 patients with mildmoderate UC received FMT via daily enemas for 5 days (22). Clinical response was observed in $78 \%$ of the patients and clinical remission was achieved in $33 \%$ within 1 week. Two thirds of the patients maintained clinical response for 1 month after the treatment. The reported adverse events were mild self-limited GI symptoms and fever.

In a recent randomized, double-blind trial in 73 adult patients with mild-moderate UC, $32 \%$ of patients achieved 
steroid free remission at 8 weeks after FMT vs. 9\% of control (autologous FMT) (23) The FMT was performed by colonoscopy followed by two enemas over 7 days.

Similar results were obtained in two other RCTs, where FMT was given by enemas or colonoscopy $(24,25)$. In a recent trial that included 41 steroid dependent adults with UC, $46 \%$ achieved clinical remission at week $24,75.6 \%$ had clinical response and $63.4 \%$ had endoscopic remission after colonoscopy administered FMT (26).

The data on the efficacy of FMT in patients with CD is limited compared to UC. A systematic review with metaanalysis of $10 \mathrm{CD}$ clinical trials and $29 \mathrm{UC}$ clinical trials reported a remission rate of $47.5 \%$ and $39.6 \%$, respectively, but there was high variability among the trials and most of them were uncontrolled (27).

Another meta-analysis of 23 cohort studies and four RCTs of CD and UC patients reported lower success rates-clinical remission rate of $28.8 \%$ and $30 \%$ for UC and $\mathrm{CD}$, respectively (28). Clinical remission did not correlate with endoscopic findings in the individuals with $\mathrm{CD}$. There was better clinical remission rate in more severe disease for both $\mathrm{UC}$ and $\mathrm{CD}$, however, there was high variability among the trials and most of them were uncontrolled.

Neither of the meta-analysis found any advantage to either route of administration nor to the feces type (frozen/fresh). For conclusion, it seems that there might be beneficial effect of FMT in patients with IBD, mainly UC. More research is needed before it can be recommended.

\section{Curcumin and resveratrol}

Curcumin is the main component of turmeric and resveratrol is a polyphenolic compound found in grapes, red wine and berries. Both of these components have been reported to have an anti-inflammatory effect.

Curcumin modulates inflammatory activity by blocking arachidonic acid cascade and NF- $\kappa \mathrm{B}$ activity, which affects CRP, TNF- $\alpha$ and IL- 1 . It downregulates genes that are involved in oxidation and fibrogenesis, regulates proliferation and activation of $\mathrm{T}$ cells, $\mathrm{B}$ cells and macrophages. It also suppresses IL-2 synthesis and inhibits neutrophils chemotaxis (29).

In a mice colitis model, subjects that were treated with DSS combined with curcumin or resveratrol had better survival rate and decreased body weight loss, diarrhea and rectal bleeding compared to the controls that were treated with DSS alone (30). Colonic TNF- $\alpha$ and IL-6 levels were significantly lower and histology was normal in the treatment groups compared to overt colitis histology in the controls.

Two multicenter randomized, placebo controlled, double-blind studies of 50 patients with mild to moderate UC and 89 patients with UC in remission on 5-ASA treatment showed significant advantage from the addition of curcumin to 5-ASA treatment compared to the 5-ASA plus placebo in clinical remission rates, clinical response, endoscopic remission rate (Mayo score $\leq 1$ ), and recurrence rate during 6 months of follow-up $(31,32)$. No adverse events were reported.

In the recent ECCO and ESPGHAN guidelines, curcumin was suggested to be used as a complementary treatment for induction of remission and maintenance in mild-moderate UC (17). Nevertheless, a recent systematic review and meta-analysis of the three UC curcumin RCT studies concluded that there is no beneficial effect to curcumin over placebo in maintaining remission (33).

Resveratrol has been demonstrated to reduce the number of Th17 cells in a mouse model of UC mouse and to increase the number of Treg cells in a dose dependent manner (34). Its anti-inflammatory effect has been demonstrated through inhibition of production of nitric oxide (NO), TNF- $\alpha$ and PGE2 and reduction of colonic epithelial cells neutrophil infiltration $(35,36)$. It was suggested that resveratrol may also have an anti-fibrotic effect (37).

Two randomized, double-blind, placebo controlled study on 50 patients with UC treated with $500 \mathrm{mg}$ resveratrol for 6 weeks found a significant reduction in markers of oxidative stress, CRP, NF- $\mathrm{kB}$ and TNF- $\alpha$ levels and a significant improvement in quality of life $(38,39)$. More clinical trials are needed to assess its efficacy and safety in IBD patients.

\section{Marijuana}

Cannabinoid receptors in the enteric nervous system influence the gut motility by decreasing gastric acid secretion and gastric and colonic emptying (40). The cannabinoids also have been demonstrated to have an antiinflammatory effect and reduction of inflammation-related pain in rats $(41,42)$.

Two randomized, double-blind, placebo controlled trials in patients with CD and one in patients with UC have failed to demonstrate any beneficial effect to the cannabis in clinical remission rate or biochemical markers, however, in one trial there was a significant clinical response in the cannabis group versus the placebo group (43-45). In 
addition, in a retrospective study in patients with $\mathrm{CD}$, there was a significant improvement in Harvey-Bradshaw index and a reduction in the number of stools per day (46).

Cannabis has also been demonstrated to improve daily activity, ability to work, health perception, reduce abdominal pain and emotional stress. It has a positive impact on the weight gain in IBD patients as well as in quality of life $(43,47)$.

Cannabis may be effective in improving symptoms such as abdominal pain and reduced appetite. It may also improve quality of life, but data suggests it has no effect on enteric inflammation. So, it can mask disease progression and may increase the risk of developing disease complication. An association between cannabis use and an increased risk for surgical intervention was demonstrated in CD patients (48).

Its chronic use, especially in the pediatric population, is of concern due to its adverse events: altered brain development, cognitive impairment with lower IQ, increased risk of psychosis disorders in susceptible patients, and addiction (49).

\section{Massage}

There is one recently published RCT that tested the effect of 30 sessions of massage therapy in patients with CD (50). There was an improvement in quality of life scores, but no effect on pain reduction was seen.

\section{Cognitive behavioral treatment (CBT)}

There is high prevalence of anxiety and depression among children with IBD (51). CBT has been demonstrated to be an effective treatment in children and adolescents with depression or anxiety disorders $(52,53)$.

Stress has been shown to induce the translocation of GI bacteria to lymphoid organs in mice (54). The parasympathetic system has an anti-inflammatory effect, while sympathetic activity has a pro-inflammatory effect. However, there is limited data to support the assumption that stress induces mucosal inflammation (55). Although stress has been shown as a contributor to mucosal damage in IBD animal models $(8,56)$, there is still limited data to support the assumption that stress induces mucosal inflammation (55).

Szigethy et al. (57) conducted an RCT of CBT on 41 adolescents with IBD. The authors concluded that CBT can lead to significant improvement in depression and global functioning compared to standard treatment.
In another large RCT that included 185 children with IBD, patients were assigned to 3 sessions of CBT vs. educational lecture that focused on nutrition, food labels and the GI system (58). There was a significant reduction in health care visits in the 12 months post intervention, improved coping ability and quality of life in the CBT group compared to the control group.

However, in contrast a recent RCT involving 70 adolescents and young adults with IBD found no advantage of CBT over standard medical care in reducing subclinical anxiety, depression or quality of life (59).

\section{Hypnotherapy}

The brain gut axis is the suggested mechanism through which hypnotherapy affects patients with IBD and IBS. There are only a few studies that have explored the efficacy of hypnotherapy in patients with IBD.

In one RCT involving 54 adults with UC, hypnotherapy was found to be effective in maintaining remission for 78 days compared to the control group (50). Two other adult studies conducted in patients with severe IBD found hypnotherapy effective in reducing the need for corticosteroid therapy, reducing disease severity and improving quality of life $(60,61)$. However, the sample size in both studies was small ( $\mathrm{n}=15$ patients in both).

Mawdsley et al. found that hypnotherapy significantly reduced substance $\mathrm{P}, \mathrm{IL}-13$ and TNF$\alpha$ levels in the rectal mucosa and reduced serum concentration of IL-6 in 17 subjects with UC (62).

In children with IBD, there is only one small study from our group (63). This study included six children-five with CD and one with UC. In this set of patients, CBT resulted in the resolution of most of the clinical symptoms and reduction of inflammatory markers.

\section{Acupuncture and moxibustion}

Acupuncture is a Chinese traditional medicine that involves placing thin needles into the patient's skin in specific points. In variations of this, an electrical current or heat is added to the treatment.

Moxibustion is another Chinese traditional medicine that involves burning mugwort cones and placing them on particular points on the skin (64).

The mechanism of acupuncture in pain relief is through several mechanisms: decreasing acetyl choline esterase levels, which are increased during inflammatory processes 
and downregulating mast cells and 5-hydroxytryptamine (5-HT) release from mast cells, thus mediating visceral afferent cells sensitization (65). Acupuncture was also found to reduce substance $\mathrm{P}$, vasoactive intestinal peptide (VIP) and neurokinin-1 receptors levels found to be overexpressed in patients with abdominal pain compared to healthy controls (65). Further, it has also been demonstrated to attenuate excitation of the colonic dorsal root ganglion, an effect that was reversed by naloxone (65).

In mouse and rat models of IBD, acupuncture has been shown to decrease the levels of TNF- $\alpha$, TNF receptors, IL-6, IL-8 and NF-אB (64).

Moxibustion improved microscopic colonic inflammation and significantly reduced the levels of several pro inflammatory cytokines in rat models of UC (66). It also up-regulated the anti-inflammatory cytokine IL-10 (67). In other work, moxibustion resulted in upregulation of tight junction proteins and decreased epithelial cell apoptosis in rat model of CD (68).

A systematic review and meta-analysis published in 2013 found that these Chinese techniques were superior to sulphasalazine (69). Nevertheless, the included trials were of low methodological quality with most of them selected clinical efficacy as an outcome, and only few used objective measurements.

In a further RCT that included 92 patients with mild to moderate $\mathrm{CD}$ there was a significant advantage to the treatment group (herb moxibustion combined with acupuncture) compared to the control group (wheat bran moxibustion combined with superficial acupuncture) (70). The two groups were treated for 12 weeks and were followed up for 24 weeks. There was a statistically significant difference in favor of the treatment group in the quality of life, increased hemoglobin level and the reduction of CRP levels in the post treatment period. Crohn's Disease Activity Index (CDAI) scores improved both in the post treatment and the follow-up period. There was no significant change in erythrocyte sedimentation rate (ESR) levels or the post treatment endoscopic evaluation. However, there was a significant difference in the relapse rate between the groups during the follow-up period in favor of the treatment group.

\section{Summary}

Based on the current data, it appears that probiotics have no effect in the setting of CD. However, VSL\#3 and E. coli Nissle 1917 have a beneficial effect as a complementary treatment for the induction of remission and maintenance in UC. VSL\#3 may also be effective in maintenance of remission in chronic pouchitis.

Current data suggests that there might be a beneficial effect for FMT in patients with IBD, however more highquality studies are needed especially in the setting of CD.

The latest published ECCO guidelines on Complementary Medicine and psychotherapy in IBD suggests curcumin as an adjuvant treatment for the induction of remission and maintenance of UC (71).

Preliminary data on the anti-inflammatory effects of resveratrol is promising, however, more clinical trials should be conducted. Cannabis may be effective in improving symptoms such as abdominal pain and reduced appetite. It may also improve quality of life, but current data indicated that it has no effect on the inflammatory process.

There is limited evidence to support the use of CBT in children or adults with IBD for the reduction of anxiety, depression and quality of life. Hypnotherapy may be an effective adjuvant therapy in improving quality of life, symptoms and inflammation in IBD, however, the current data is limited and more studies are needed. There is insufficient data to support the use of acupuncture or moxibustion as complementary treatments in IBD.

\section{Acknowledgments}

None.

\section{Footnote}

Conflicts of Interest: The authors have no conflicts of interest to declare.

Ethical Statement: The authors are accountable for all aspects of the work in ensuring that questions related to the accuracy or integrity of any part of the work are appropriately investigated and resolved.

\section{References}

1. Lin SC, Cheifetz AS. The Use of Complementary and Alternative Medicine in Patients With Inflammatory Bowel Disease. Gastroenterol Hepatol (N Y) 2018;14:415-25.

2. Abraham C, Cho JH. Inflammatory bowel disease. N Engl J Med 2009;361:2066-78.

3. Manichanh C, Rigottier-Gois L, Bonnaud E, et al. Reduced diversity of faecal microbiota in Crohn's disease revealed by a 
metagenomic approach. Gut 2006;55:205-11.

4. Gevers D, Kugathasan S, Denson LA, et al. The treatment-naive microbiome in new-onset Crohn's disease. Cell Host Microbe 2014;15:382-92.

5. Zuo T, Ng SC. The Gut Microbiota in the Pathogenesis and Therapeutics of Inflammatory Bowel Disease. Front Microbiol 2018;9:2247.

6. Coqueiro AY, Raizel R, Bonvini A, et al. Probiotics for inflammatory bowel diseases: a promising adjuvant treatment. Int J Food Sci Nutr 2019;70:20-9.

7. Sivananthan K, Petersen AM. Review of Saccharomyces boulardii as a treatment option in IBD. Immunopharmacol Immunotoxicol 2018;40:465-75.

8. Derwa Y, Gracie DJ, Hamlin PJ, et al. Systematic review with meta-analysis: the efficacy of probiotics in inflammatory bowel disease. Aliment Pharmacol Ther 2017;46:389-400.

9. Fedorak RN, Feagan BG, Hotte N, et al. The probiotic VSL\#3 has anti-inflammatory effects and could reduce endoscopic recurrence after surgery for Crohn's disease. Clin Gastroenterol Hepatol 2015;13:928-35.e2.

10. Miele E, Pascarella F, Giannetti E, et al. Effect of a probiotic preparation (VSL\#3) on induction and maintenance of remission in children with ulcerative colitis. Am J Gastroenterol 2009;104:437-43.

11. Huynh HQ, deBruyn J, Guan L, et al. Probiotic preparation VSL\#3 induces remission in children with mild to moderate acute ulcerative colitis: a pilot study. Inflamm Bowel Dis 2009;15:760-8.

12. Gionchetti P, Rizzello F, Venturi A, et al. Oral bacteriotherapy as maintenance treatment in patients with chronic pouchitis: a double-blind, placebo-controlled trial. Gastroenterology 2000;119:305-9.

13. Mimura T, Rizzello F, Helwig U, et al. Once daily high dose probiotic therapy (VSL\#3) for maintaining remission in recurrent or refractory pouchitis. Gut 2004;53:108-14.

14. Lin PW, Myers LE, Ray L, et al. Lactobacillus rhamnosus blocks inflammatory signaling in vivo via reactive oxygen species generation. Free Radic Biol Med 2009;47:1205-11.

15. Pagnini C, Corleto VD, Martorelli M, et al. Mucosal adhesion and anti-inflammatory effects of Lactobacillus rhamnosus GG in the human colonic mucosa: A proof-ofconcept study. World J Gastroenterol 2018;24:4652-62.

16. Losurdo G, Iannone A, Contaldo A, et al. Escherichia coli Nissle 1917 in Ulcerative Colitis Treatment: Systematic Review and Meta-analysis. J Gastrointestin Liver Dis 2015;24:499-505.

17. Turner D, Ruemmele FM, Orlanski-Meyer E, et al.
Management of Paediatric Ulcerative Colitis, Part 1: Ambulatory Care-An Evidence-based Guideline From European Crohn's and Colitis Organization and European Society of Paediatric Gastroenterology, Hepatology and Nutrition. J Pediatr Gastroenterol Nutr 2018;67:257-91.

18. Weingarden AR, Vaughn BP. Intestinal microbiota, fecal microbiota transplantation, and inflammatory bowel disease. Gut Microbes 2017;8:238-52.

19. Couturier-Maillard A, Secher T, Rehman A, et al. NOD2mediated dysbiosis predisposes mice to transmissible colitis and colorectal cancer. J Clin Invest 2013;123:700-11.

20. Rossen NG, Fuentes S, van der Spek MJ, et al. Findings From a Randomized Controlled Trial of Fecal Transplantation for Patients With Ulcerative Colitis. Gastroenterology 2015;149:110-118.e4.

21. Suskind DL, Singh N, Nielson H, et al. Fecal microbial transplant via nasogastric tube for active pediatric ulcerative colitis. J Pediatr Gastroenterol Nutr 2015;60:27-9.

22. Kunde S, Pham A, Bonczyk S, et al. Safety, tolerability, and clinical response after fecal transplantation in children and young adults with ulcerative colitis. J Pediatr Gastroenterol Nutr 2013;56:597-601.

23. Costello SP, Hughes PA, Waters O, et al. Effect of Fecal Microbiota Transplantation on 8-Week Remission in Patients With Ulcerative Colitis: A Randomized Clinical Trial. JAMA 2019;321:156-64.

24. Moayyedi P, Surette MG, Kim PT, et al. Fecal Microbiota Transplantation Induces Remission in Patients With Active Ulcerative Colitis in a Randomized Controlled Trial. Gastroenterology 2015;149:102-109.e6.

25. Paramsothy S, Kamm MA, Kaakoush NO, et al. Multidonor intensive faecal microbiota transplantation for active ulcerative colitis: a randomised placebo-controlled trial. Lancet 2017;389:1218-28.

26. Sood A, Mahajan R, Juyal G, et al. Efficacy of fecal microbiota therapy in steroid dependent ulcerative colitis: a real world intention-to-treat analysis. Intest Res 2019;17:78-86.

27. Lai CY, Sung J, Cheng F, et al. Systematic review with meta-analysis: review of donor features, procedures and outcomes in 168 clinical studies of faecal microbiota transplantation. Aliment Pharmacol Ther 2019;49:354-63.

28. Fang H, Fu L, Wang J. Protocol for Fecal Microbiota Transplantation in Inflammatory Bowel Disease: A Systematic Review and Meta-Analysis. Biomed Res Int 2018;2018:8941340.

29. Ali T, Shakir F, Morton J. Curcumin and inflammatory 
bowel disease: biological mechanisms and clinical implication. Digestion 2012;85:249-55.

30. Zhang L, Xue H, Zhao G, et al. Curcumin and resveratrol suppress dextran sulfate sodiuminduced colitis in mice. Mol Med Rep 2019;19:3053-60.

31. Hanai H, Iida T, Takeuchi K, et al. Curcumin maintenance therapy for ulcerative colitis: randomized, multicenter, double-blind, placebo-controlled trial. Clin Gastroenterol Hepatol 2006;4:1502-6.

32. Lang A, Salomon N, Wu JC, et al. Curcumin in Combination With Mesalamine Induces Remission in Patients With Mild-to-Moderate Ulcerative Colitis in a Randomized Controlled Trial. Clin Gastroenterol Hepatol 2015;13:1444-9.e1.

33. Grammatikopoulou MG, Gkiouras K, Theodoridis X, et al. Oral Adjuvant Curcumin Therapy for Attaining Clinical Remission in Ulcerative Colitis: A Systematic Review and Meta-Analysis of Randomized Controlled Trials. Nutrients 2018. doi: 10.3390/nu10111737.

34. Yao J, Wei C, Wang JY, et al. Effect of resveratrol on Treg/ Th17 signaling and ulcerative colitis treatment in mice. World J Gastroenterol 2015;21:6572-81.

35. Kim MH, Son YJ, Lee SY, et al. JAK2-targeted anti-inflammatory effect of a resveratrol derivative 2,4-dihydroxy-N-(4-hydroxyphenyl)benzamide. Biochem Pharmacol 2013;86:1747-61.

36. Mayangsari Y, Suzuki T. Resveratrol Ameliorates Intestinal Barrier Defects and Inflammation in Colitic Mice and Intestinal Cells. J Agric Food Chem 2018;66:12666-74.

37. Li P, Liang ML, Zhu Y, et al. Resveratrol inhibits collagen I synthesis by suppressing IGF-1R activation in intestinal fibroblasts. World J Gastroenterol 2014;20:4648-61.

38. Samsamikor M, Daryani NE, Asl PR, et al. Resveratrol Supplementation and Oxidative/Anti-Oxidative Status in Patients with Ulcerative Colitis: A Randomized, DoubleBlind, Placebo-controlled Pilot Study. Arch Med Res 2016;47:304-9.

39. Samsami-Kor M, Daryani NE, Asl PR, et al. AntiInflammatory Effects of Resveratrol in Patients with Ulcerative Colitis: A Randomized, Double-Blind, Placebocontrolled Pilot Study. Arch Med Res 2015;46:280-5.

40. Quezada SM, Cross RK. Cannabis and Turmeric as Complementary Treatments for IBD and Other Digestive Diseases. Curr Gastroenterol Rep 2019;21:2.

41. Costa B, Trovato AE, Comelli F, et al. The nonpsychoactive cannabis constituent cannabidiol is an orally effective therapeutic agent in rat chronic inflammatory and neuropathic pain. Eur J Pharmacol 2007;556:75-83.
42. Sumariwalla PF, Gallily R, Tchilibon S, et al. A novel synthetic, nonpsychoactive cannabinoid acid (HU-320) with antiinflammatory properties in murine collageninduced arthritis. Arthritis Rheum 2004;50:985-98.

43. Irving PM, Iqbal T, Nwokolo C, et al. A Randomized, Double-blind, Placebo-controlled, Parallel-group, Pilot Study of Cannabidiol-rich Botanical Extract in the Symptomatic Treatment of Ulcerative Colitis. Inflamm Bowel Dis 2018;24:714-24.

44. Naftali T, Bar-Lev Schleider L, Dotan I, et al. Cannabis induces a clinical response in patients with Crohn's disease: a prospective placebo-controlled study. Clin Gastroenterol Hepatol 2013;11:1276-80.e1.

45. Naftali T, Mechulam R, Marii A, et al. Low-Dose Cannabidiol Is Safe but Not Effective in the Treatment for Crohn's Disease, a Randomized Controlled Trial. Dig Dis Sci 2017;62:1615-20.

46. Naftali T, Lev LB, Yablecovitch D, et al. Treatment of Crohn's disease with cannabis: an observational study. Isr Med Assoc J 2011;13:455-8. Erratum in: Isr Med Assoc J. 2011 Sep;13(9):582. Yablekovitz, Doron [corrected to Yablecovitch, Doron].

47. Lahat A, Lang A, Ben-Horin S. Impact of cannabis treatment on the quality of life, weight and clinical disease activity in inflammatory bowel disease patients: a pilot prospective study. Digestion 2012;85:1-8.

48. Storr M, Devlin S, Kaplan GG, et al. Cannabis use provides symptom relief in patients with inflammatory bowel disease but is associated with worse disease prognosis in patients with Crohn's disease. Inflamm Bowel Dis 2014;20:472-80.

49. Volkow ND, Compton WM, Weiss SR. Adverse health effects of marijuana use. N Engl J Med 2014;371:879.

50. Espí-López GV, Ingles M, Soliva-Cazaban I, et al. Effect of the soft-tissue techniques in the quality of life in patients with Crohn's disease: A randomized controlled trial. Medicine (Baltimore) 2018;97:e13811.

51. Yeh AM, Wren A, Golianu B. Mind-Body Interventions for Pediatric Inflammatory Bowel Disease. Children (Basel) 2017. doi: 10.3390/children4040022.

52. Oud M, de Winter L, Vermeulen-Smit E, et al. Effectiveness of CBT for children and adolescents with depression: A systematic review and meta-regression analysis. Eur Psychiatry 2019;57:33-45.

53. Villabø MA, Narayanan M, Compton SN, et al. Cognitivebehavioral therapy for youth anxiety: An effectiveness evaluation in community practice. J Consult Clin Psychol 2018;86:751-64. 
54. Bailey MT, Engler H, Sheridan JF. Stress induces the translocation of cutaneous and gastrointestinal microflora to secondary lymphoid organs of C57BL/6 mice. J Neuroimmunol 2006;171:29-37.

55. Bernstein CN. The Brain-Gut Axis and Stress in Inflammatory Bowel Disease. Gastroenterol Clin North Am 2017;46:839-46.

56. Bonaz BL, Bernstein CN. Brain-gut interactions in inflammatory bowel disease. Gastroenterology 2013;144:36-49.

57. Szigethy E, Kenney E, Carpenter J, et al. Cognitivebehavioral therapy for adolescents with inflammatory bowel disease and subsyndromal depression. J Am Acad Child Adolesc Psychiatry 2007;46:1290-8.

58. Levy RL, van Tilburg MA, Langer SL, et al. Effects of a Cognitive Behavioral Therapy Intervention Trial to Improve Disease Outcomes in Children with Inflammatory Bowel Disease. Inflamm Bowel Dis 2016;22:2134-48.

59. Stapersma L, van den Brink G, van der Ende J, et al. Effectiveness of Disease-Specific Cognitive Behavioral Therapy on Anxiety, Depression, and Quality of Life in Youth With Inflammatory Bowel Disease: A Randomized Controlled Trial. J Pediatr Psychol 2018;43:967-80.

60. Keefer L, Kiebles JL, Kwiatek MA, et al. The potential role of a self-management intervention for ulcerative colitis: a brief report from the ulcerative colitis hypnotherapy trial. Biol Res Nurs 2012;14:71-7.

61. Miller V, Whorwell PJ. Treatment of inflammatory bowel disease: a role for hypnotherapy? Int J Clin Exp Hypn 2008;56:306-17.

62. Mawdsley JE, Jenkins DG, Macey MG, et al. The effect of hypnosis on systemic and rectal mucosal measures of inflammation in ulcerative colitis. Am J Gastroenterol 2008;103:1460-9.

Cite this article as: Magen R, Shaoul R. Alternative \& complementary treatment for pediatric inflammatory bowel disease. Transl Pediatr 2019;8(5):428-435. doi: 10.21037/ tp.2019.09.02
63. Shaoul R, Sukhotnik I, Mogilner J. Hypnosis as an adjuvant treatment for children with inflammatory bowel disease. J Dev Behav Pediatr 2009;30:268.

64. Stein DJ. Massage Acupuncture, Moxibustion, and Other Forms of Complementary and Alternative Medicine in Inflammatory Bowel Disease. Gastroenterol Clin North Am 2017;46:875-80.

65. Zhang F, Wu L, Zhao J, et al. Neurobiological Mechanism of Acupuncture for Relieving Visceral Pain of Gastrointestinal Origin. Gastroenterol Res Pract 2017;2017:5687496.

66. Zhang D, Ren YB, Wei K, et al. Herb-partitioned moxibustion alleviates colon injuries in ulcerative colitis rats. World J Gastroenterol 2018;24:3384-97.

67. Ma TM, Han Y, Ma XD, et al. Influence of moxibustion with different duration on colonic epithelial structure, serum inflammatory cytokines, and intestinal mucosa inflammatory cell signal transduction pathways. Zhen $\mathrm{Ci}$ Yan Jiu 2014;39:20-6.

68. Bao CH, Wu LY, Shi Y, et al. Moxibustion down-regulates colonic epithelial cell apoptosis and repairs tight junctions in rats with Crohn's disease. World J Gastroenterol 2011;17:4960-70.

69. Ji J, Lu Y, Liu H, et al. Acupuncture and moxibustion for inflammatory bowel diseases: a systematic review and meta-analysis of randomized controlled trials. Evid Based Complement Alternat Med 2013;2013:158352.

70. Bao CH, Zhao JM, Liu HR, et al. Randomized controlled trial: moxibustion and acupuncture for the treatment of Crohn's disease. World J Gastroenterol 2014;20:11000-11.

71. Torres J, Ellul P, Langhorst J, et al. European Crohn's and Colitis Organisation Topical Review on Complementary Medicine and Psychotherapy in Inflammatory Bowel Disease. J Crohns Colitis 2019;13:673-85e. 